\title{
Three-Dimensional Numerical Simulation on Marangoni Convection in a Sessile Water Droplet Evaporating in its Vapor at Low Pressure
}

\author{
Yu Zhang ${ }^{1}$. You-Rong $\mathrm{Li}^{1}$ • Jia-Jia Yu ${ }^{1}$ - Qiu-Sheng Liu ${ }^{2}$
}

Received: 8 November 2018 / Accepted: 27 February 2019

(C) Springer Nature B.V. 2019

\begin{abstract}
In order to understand the effect of Marangoni convection on the evaporation rate and the flow pattern, we performed a series of three-dimensional numerical simulations on evaporation process of sessile water droplet by introducing a kinetic model. Substrate temperature and the ratio of vapor pressure varied from $299 \mathrm{~K}$ to $308 \mathrm{~K}$ and from 0.92 to 0.99 , respectively. The variation range of contact angle $\theta$ was between $9.15^{\circ}$ and $120^{\circ}$. Results show that the axisymmetric ring-shape temperature distribution on droplet free surface transforms into a serrated temperature distribution because of the enhancing Marangoni convection with the increase of substrate temperature. In addition, with the decrease of vapor pressure, the total evaporation rate on free surface will be increased and the Marangoni effect will be enhanced. The flow pattern is axisymmetric at $90^{\circ} \leq \theta \leq 120^{\circ}$ when the substrate temperature is fixed. However, it shifts to a multi-cellular pattern with the decrease of contact angle as a result of the competition between Marangoni flow and the evaporation. Additionally, the total evaporation rate increases with the increase of contact angle when contact angle is above $60^{\circ}$, but when the contact angle is below $60^{\circ}$, the trend is reverted. The temperature distribution becomes distinct at a small contact angle.
\end{abstract}

Keywords Sessile droplet $\cdot$ Evaporation $\cdot$ Marangoni convection $\cdot$ Kinetic model $\cdot$ Numerical simulation

\author{
Nomenclature \\ a thermal diffusivity, $\mathrm{m}^{2} / \mathrm{s}$ \\ $g \quad$ gravity acceleration, $\mathrm{m} / \mathrm{s}^{2}$ \\ $h \quad$ height of the droplet, $\mathrm{m}$ \\ $H_{\mathrm{L}} \quad$ latent heat of vaporization, $\mathrm{J} / \mathrm{kg}$ \\ $J \quad$ evaporation rate, $\mathrm{kg} /\left(\mathrm{m}^{2} \cdot \mathrm{s}\right)$ \\ $k_{1} \quad$ thermal conductivity, $\mathrm{W} /(\mathrm{m} \cdot \mathrm{K})$ \\ $l_{c} \quad$ capillary length, $\mathrm{m}$ \\ $M \quad$ molar mass of the evaporating liquid, $\mathrm{g} / \mathrm{mol}$ \\ $P \quad$ pressure, $\mathrm{Pa}$ \\ $q_{\mathrm{m}} \quad$ evaporation flux, $\mathrm{kg} / \mathrm{s}$ \\ $r \quad$ contact radius, $\mathrm{m}$ \\ $R \quad$ universal gas constant, $\mathrm{J} /(\mathrm{mol} \cdot \mathrm{K})$
}

You-Rong Li

liyourong@cqu.edu.cn

Key Laboratory of Low-grade Energy Utilization Technologies and Systems of Ministry of Education, School of Energy and Power Engineering, Chongqing University, Chongqing 400044, China

2 Key Laboratory of Microgravity (National Microgravity Laboratory), Institute of Mechanics, Chinese Academy of Sciences, Beijing 100190, China

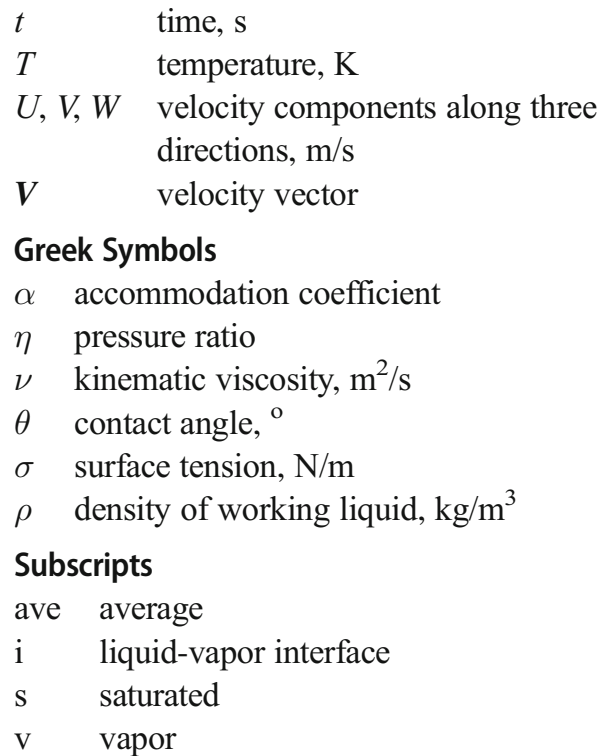

\section{Introduction}

The sessile droplet evaporation has a wide range of practical applications, such as spray cooling (Sodtke and Stephan 2007; 
Cheng et al. 2011), surface modification (Haschke et al. 2007; Pericet-Camara et al. 2010), ink-jet printing (Park and Moon 2006) and food preservation (D'Agaro et al. 2006). The socalled "coffee ring" phenomena, which occurs in particleladen sessile droplet, has been used as the basis of the highthroughout DNA mapping technology and disease diagnose (Deegan et al. 1997; Sefiane 2010). Nevertheless, most of the existing researches focus mainly on the sessile droplet evaporating in the open air but few investigations concern about the sessile droplet evaporation in its low-pressure vapor environment, which is of great significance in flash distillation, desalination and vacuum cooling (Liu and Mi 2014). For instance, vacuum flash evaporation cooling (VFEC) including the process of evaporation in vapor is an advanced technique, which has been used in the thermal control system of NASA Orion Crew Exploration Vehicle spacecraft (Cheng et al. 2016).

Evaporation will decrease the interfacial temperature and lead to the temperature inhomogeneity in an evaporating sessile droplet, which is called as evaporative cooling effect. $\mathrm{Xu}$ and $\mathrm{Ma}$ (2015) introduced a dimensionless evaporative cooling number to signify the intensify of the evaporative cooling effect and found that total evaporation flux and evaporation rate increased when the evaporative cooling number increased. Wang et al. (2016) also used the evaporative cooling number to discuss the evaporative cooling effect on evaporation rate at different contact angles. The result illustrated that the total evaporation rate increased with the contact angle at a small evaporative cooling number but decreased at a large evaporative cooling number. Furthermore, Girard et al. (2006; Girard and Antoni 2008) studied the Marangoni flow and Stefan flow during the evaporation of sessile droplet at different substrate temperature and different heating substrate size. It was found that when the heating substrate size was no less than droplet radius, the convection led to a downstream flow near the symmetry axis of the droplet, but the upstream flow was observed when heating substrate size was smaller than radius. Barash (2016) numerically investigated the Marangoni convection inside the evaporating droplet in a wide range of contact angles. Moreover, an analytical approach was developed to describe the single-vortex Marangoni flow appropriately. Furthermore, Brutin et al. (2010) and Zhu et al. (2010) reported the experimental observation on the evaporation of the sessile drop in microgravity and certified the different surface temperature distribution.

In order to simplify the model, researchers normally exclude one of the Marangoni flow and the evaporative cooling effect (Dunn et al. 2009b; Picknett and Bexon 1977; Hu and Larson 2002) or neglect both of them. For instance, Dunn et al. (2009a) investigated the influence of substrate conductivity on droplet evaporation by using Newton cooling law to emulate the heat transfer caused by evaporation. $\mathrm{Hu}$ and Larson (2005) analyzed the flow field induced by evaporation. They ignored both Marangoni flow and evaporative cooling, so the results were only accurate in some certain cases. Xu and Luo (2007) certified experimentally the existence of Marangoni flow in evaporating deionized water droplets by using fluorescent nanoparticles. Furthermore, Ghasemi and Ward (2010) found experimentally that the evaporation mainly occured in the region close to the contact line, and most of energy provided for evaporation in this region was from Marangoni convection. According to the research performed by Yang et al. (2014), the isothermal model without the evaporative cooling effect can't predict the interface temperature precisely. Therefore, it is inappropriate to neglect the Marangoni flow or evaporative cooling effect of the sessile droplet.

The temperature of the solid substrate is a key factor influencing evaporation process of sessile droplet. Crafton

Fig. 1 Physical model

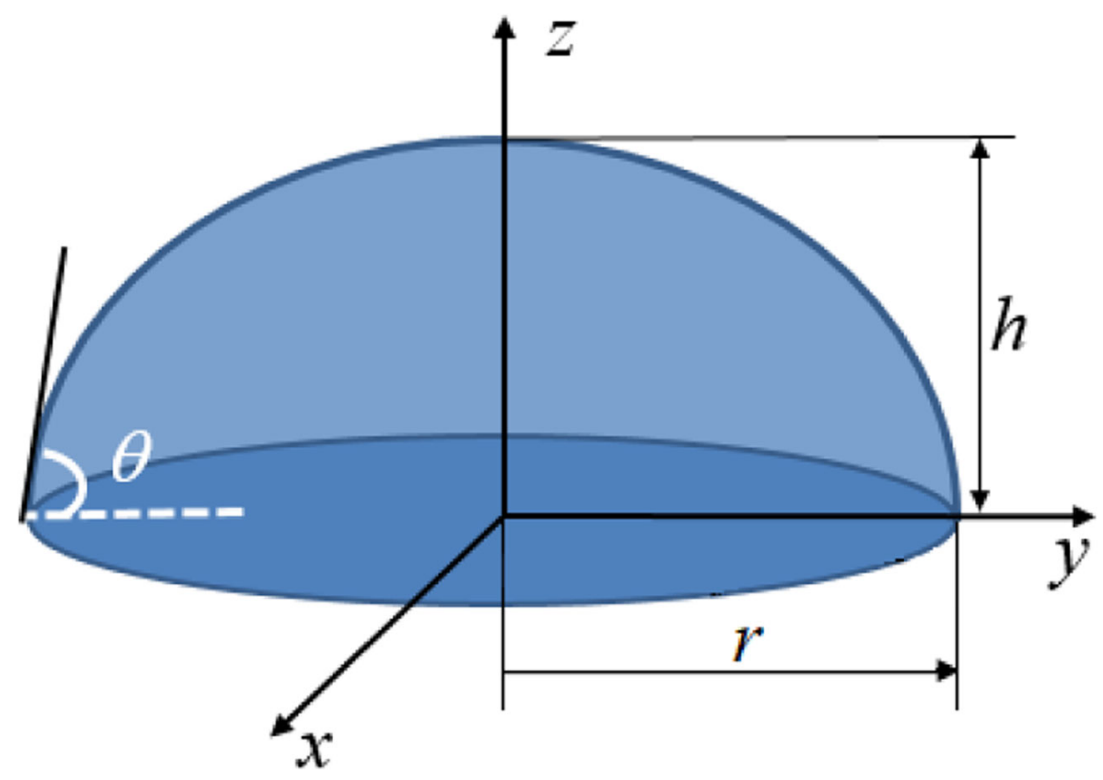


Table 1 Grid sensitivity analysis at $h=1 \mathrm{~mm}, T_{w}=302 \mathrm{~K}$ and $\eta=1$

\begin{tabular}{lllll}
\hline Mesh No. & Number of cells & $\begin{array}{l}\text { The lowest temperature } \\
\text { on free surface (K) }\end{array}$ & $\begin{array}{l}\text { The total heat flux through } \\
\text { the free surface (W) }\end{array}$ & $\begin{array}{l}\text { Max relative } \\
\text { deviation (\%) }\end{array}$ \\
\hline 1 & 88,556 & 298.1983 & -0.2482 & 2.31 \\
2 & 182,827 & 298.1985 & -0.2426 & - \\
3 & 287,553 & 298.1987 & -0.2375 & 2.10 \\
4 & 340,605 & 298.1986 & -0.2383 & 1.77 \\
\hline
\end{tabular}

and Black (2004) found experimentally that the evaporation rate increased as the substrate temperature increased. Chen et al. (2017) also certified that the evaporation rate followed power-law scaling with substrate temperature. Girard et al. (2008) discussed the influence of substrate temperature, droplet size and relative humidity simultaneously. An empirical law was obtained for evaporating time as a function of these three variables. Besides, Sefiane et al. (2007) analyzed the influence of ambient pressure on sessile droplet evaporation and found that evaporation rate would be increased by reducing ambient pressure.

To the best of our knowledge, the most of numerical researches focus on the droplet evaporation in the open air using a diffusion model (Sefiane and Bennacer 2011; Chen et al. 2018; Kelly 2013). In this paper, the kinetic model is used to simulate the evaporating droplet in its vapor. The influences of substrate temperature, vapor pressure and contact angle on temperature distribution, flow pattern and evaporation mass flux are discussed. For a droplet evaporating in its vapor, the diffusion model is not suitable anymore. So the HertzKnudsen-Schrage equation from kinetic theory is introduced to obtain the evaporation mass flux. The parameters in the equation can be easily assured by the experiment. Additionally, compared with the two-dimensional models, we can observe the variation of flow and temperature fields on the free surface.

\section{Problem Formulation}

The sketch of the physical model is provided in Fig. 1. The contact line keeps pinned during the evaporation. On the liquid-solid interface, a no-slip boundary condition is used. Considering the small size of the droplet and the weak flow of vapor in the low-pressure environment, we neglect the influence of vapor on the flow and temperature fields of an evaporating droplet. The thermal conductivity of solid substrate is large enough compared with the conductivities of vapor and liquid, so the substrate is set as isothermal. The temperature of solid substrate is constant.

The capillary length is defined as $l_{c}=\sqrt{\sigma / \rho g}$. The value for water is equal to $2.7 \mathrm{~mm}$ (Semenov et al. 2012a). Because the height and contact radius of the sessile water droplet are smaller than capillary length, the shape will maintain a spherical cap.

Several assumptions are introduced to simplify the model. (1) Liquid droplet is uncompressible Newtonian fluid, and all the thermophysical properties are constant except that surface tension varies linearly with temperature. (2) Liquid-vapor interface is regarded as indeformable and contact line is pinned during the evaporation. (3) Because the droplet size is much larger than $10^{-7} \mathrm{~m}$, the Kelvin effect caused by curvature of the droplet is negligible (Semenov et al. 2012b).

Based on assumptions mentioned above, the continuity equation, Navier-Strokes equations and the energy equation can be expressed by following forms:

$\nabla \cdot \mathrm{V}=0$

$\frac{\partial \mathrm{V}}{\partial t}+(\mathrm{V} \cdot \nabla) \mathrm{V}=-\frac{1}{\rho} \nabla P+\nu \nabla^{2} \mathrm{~V}$

$\frac{\partial T}{\partial t}+(\mathrm{V} \cdot \nabla) \cdot T=a \nabla^{2} T$

At the bottom of the droplet, the temperature of isothermal substrate is constant.

$T=T_{\mathrm{w}}$

On the solid-liquid interface, the no-slip and no-penetration boundary conditions are adopted,

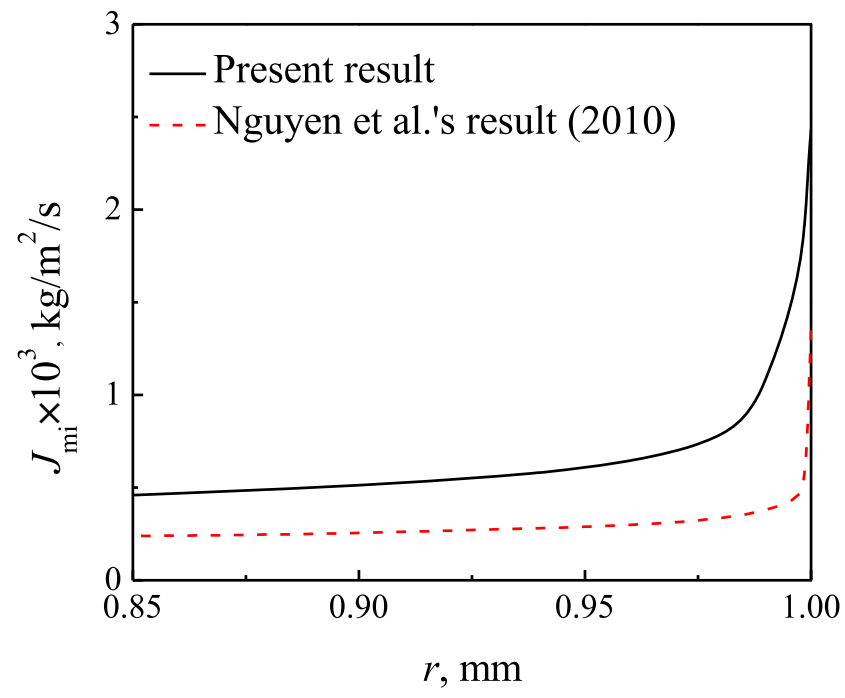

Fig. 2 The comparison of the evaporating rate 
$U=V=W=0$

The evaporating rate $J$ is calculated by Hertz-KnudsenSchrage formula (Persad and Ward 2016)

$J=\frac{2 \alpha}{2-\alpha} \sqrt{\frac{M}{2 \pi R}}\left(\frac{P_{s}\left(T_{i}\right)}{\sqrt{T_{i}}}-\frac{P_{v}}{\sqrt{T_{v}}}\right)$

Where $\alpha$ is the accommodation coefficient and is set as 0.04 based on the work of Marek and Straub (2001). $P_{s}$ is the saturation pressure of vapor at interface temperature $T_{\mathrm{i}}$, which can be determined by following fitting formula (Unsworth 1974):
$P_{S}\left(T_{i}\right)=610.7 \times 10^{\frac{7.5\left(T_{i}-273.15\right)}{\left(T_{i}-35.85\right)}}$

The energy balance on the droplet surface can be expressed as follow:

$-k_{l} \nabla T \cdot \mathrm{n}=H_{L} \cdot J$

The working fluid is water. The temperature of the substrate varies from $299 \mathrm{~K}$ to $308 \mathrm{~K}$. The contact radius of sessile droplet is fixed at $2.5 \mathrm{~mm}$, but the variation range of contact angle is between $9.15^{\circ}$ and $120^{\circ}$. The pressure ratio $\eta$ is defined as the ratio of actual vapor pressure to saturation vapor

Fig. 3 The temperature

distribution on the free surface (top) and inner slice (middle), the velocity vector field on the inner slice (bottom)

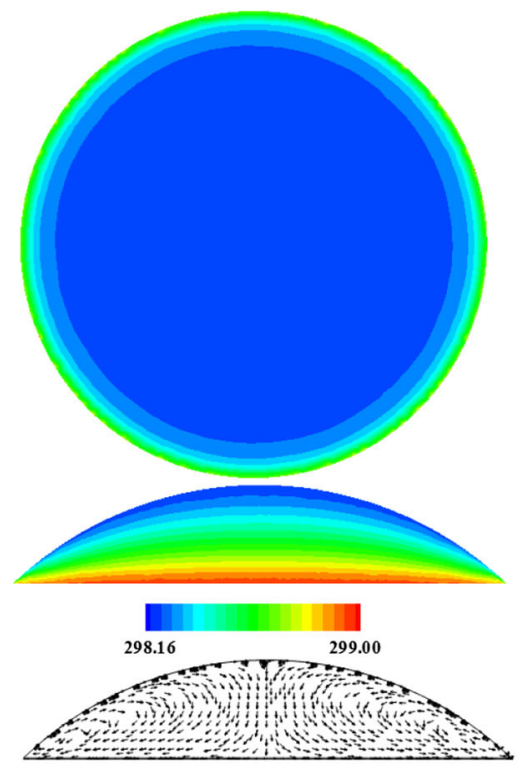

(a) $T_{\mathrm{w}}=299 \mathrm{~K}$

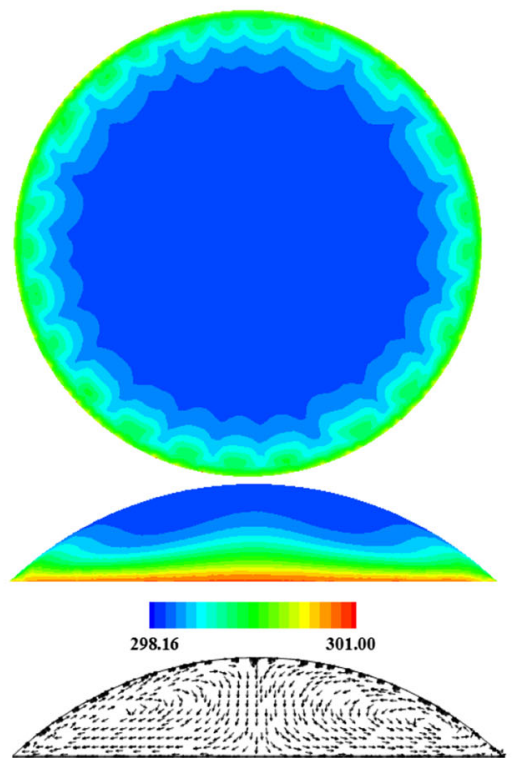

(c) $T_{\mathrm{w}}=301 \mathrm{~K}$

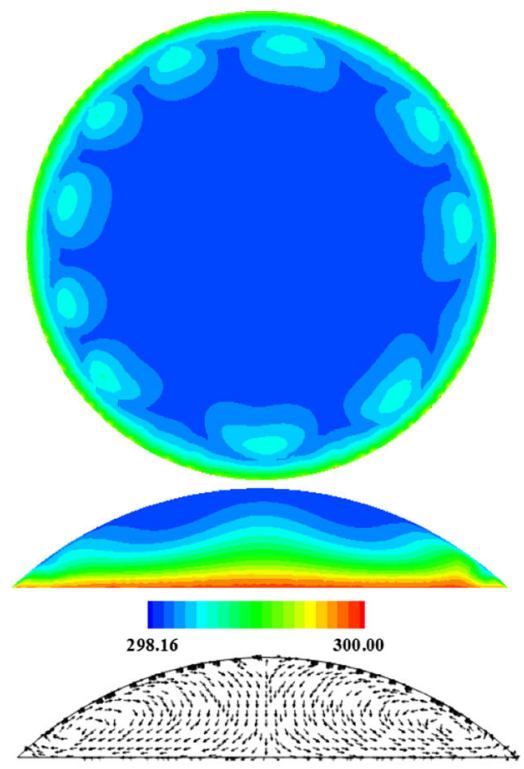

(b) $T_{\mathrm{w}}=300 \mathrm{~K}$

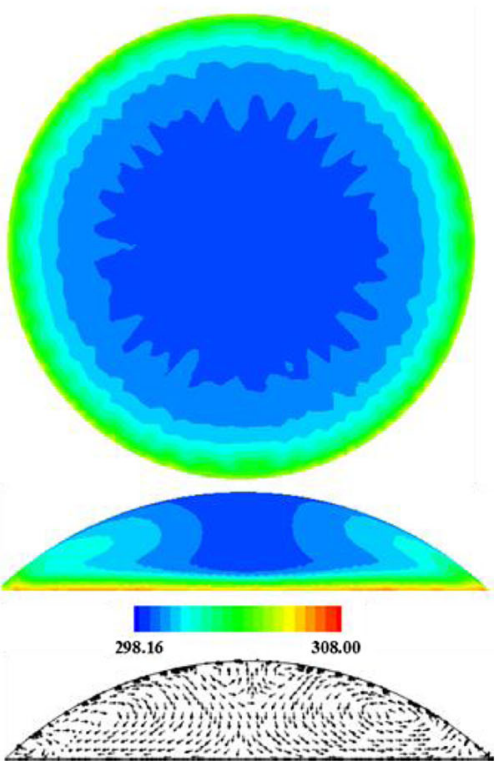

(d) $T_{\mathrm{w}}=308 \mathrm{~K}$ 
pressure at the temperature of surrounding vapor. It ranges from 0.92 to 0.99 .

\section{Numerical Procedure and Validation}

The numerical simulation is completed by commercial software Fluent 6.3.26 based on finite volume method. A Userdefined Function is loaded to calculate the heat flux through liquid-vapor interface. The QUICK scheme is employed to treat the convective terms while diffusion terms are handled by central difference approximation. The SIMPLEC algorism is chosen to solve the pressure-velocity coupling equations. In the process of iteration, when the maximum relative error of velocity and temperature is no more than $10^{-4}$, we think the solution is convergent.

The unstructured grid is used. We choose the lowest temperature and the total heat flux on the droplet surface as key variables to test the grid sensitivity, as shown in Table 1. It can be found that the maximum relative deviation between Mesh No. 2 and other sets of mesh does not exceed 3\%. Therefore, the Mesh No. 2 is selected for further simulations.

In order to verify the numerical method, we calculated the total evaporation rate on free surface at $T_{\mathrm{w}}=298 \mathrm{~K}, \eta=0.92$ and $\theta=43.6^{\circ}$, and compared with the experimental result at the same conditions. Experimental procedure is the similar with the work of Ye et al. (2018). It was found that the deviation of the evaporation rate of the water droplet between simulation and experimental results is less than $15.6 \%$. Also, we compare the result obtained by a kinetic model with Nguyen's result (Nguyen et al. 2010) by a diffusion model. It can be found that there exists the same variation trend of the evaporating rate distribution with the result from HertzKnudsen-Schrage equation. However, because of the larger evaporating resistance in an open-air surrounding than in a pure-vapor environment, the evaporating rate in an open-air surrounding is much less than that in a pure-vapor environment, as shown in Fig. 2.

\section{Results and Discussion}

\section{The Influence of Substrate Temperature}

In this section, temperature of the saturation vapor is fixed at $T_{\mathrm{v}}=298 \mathrm{~K}$, and corresponding saturated pressure is $P_{\mathrm{v}}=$ $3168 \mathrm{~Pa}$. The temperature difference between substrate and vapor has an important effect on temperature and flow fields in the droplet. As shown in Fig. 3, as the substrate temperature varies between $299 \mathrm{~K}$ and $308 \mathrm{~K}$, the temperature distribution on the droplet surface evolves from the ring shape to the petal shape, then the serrated shape. When substrate temperature increases to $300 \mathrm{~K}$, torus rolls emerge near the contact line.

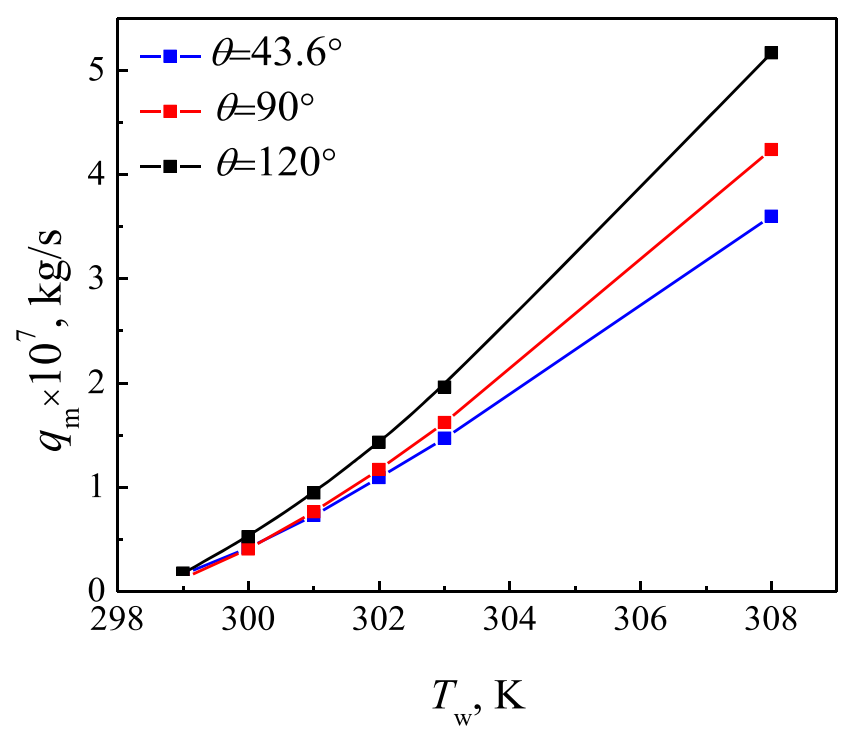

Fig. 4 Variation of total evaporation mass flux with the substrate temperature at $\eta=1$

The similar flow pattern was also observed in Semenov et al.'s work (2017). They reckoned that the appearance of torus rolls near the contact line should be attributed to the MarangoniBenard instability, which is resulted from a vertical temperature gradient in the liquid bulk as well as a tangential one along the droplet surface near the contact line. The evaporation decreases the interfacial temperature but the region close to substrate has a high temperature because of the heat conduction from substrate, which is recognized as the main reason for these temperature gradients. Because there is no basic shear flow along the tangential direction, this instability could not be ascribed to hydrothermal wave instability.

The temperature of the droplet apex is lower than one at its edge, so the surface tension at the apex is larger than that at contact line, which drives the Marangoni convection from the contact line region towards apex. The fluid gathering at the droplet apex flows towards the bottom of the droplet, thus a

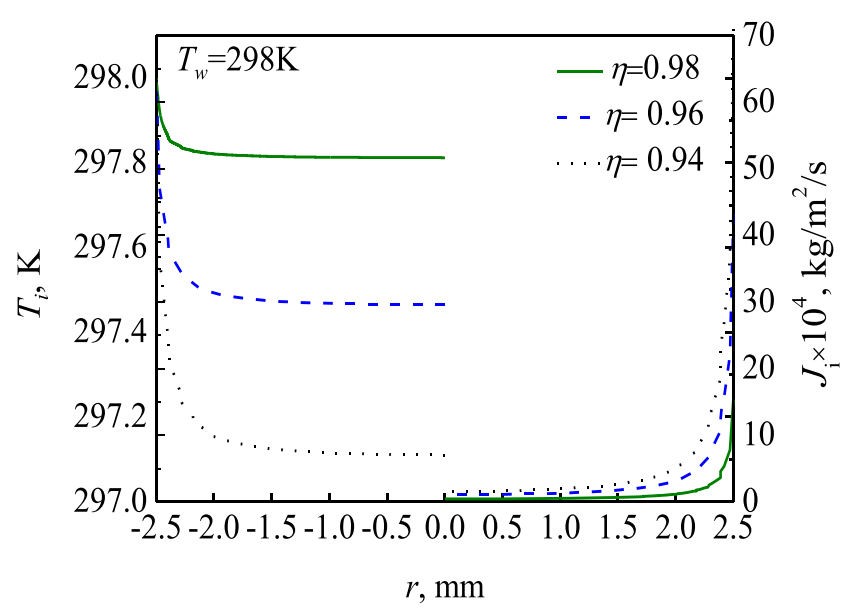

Fig. 5 The local temperature and evaporation rate along the same curve on free surface 
Fig. 6 The surface temperature distribution when $\eta=0.93$ (left) and 0.92 (right)
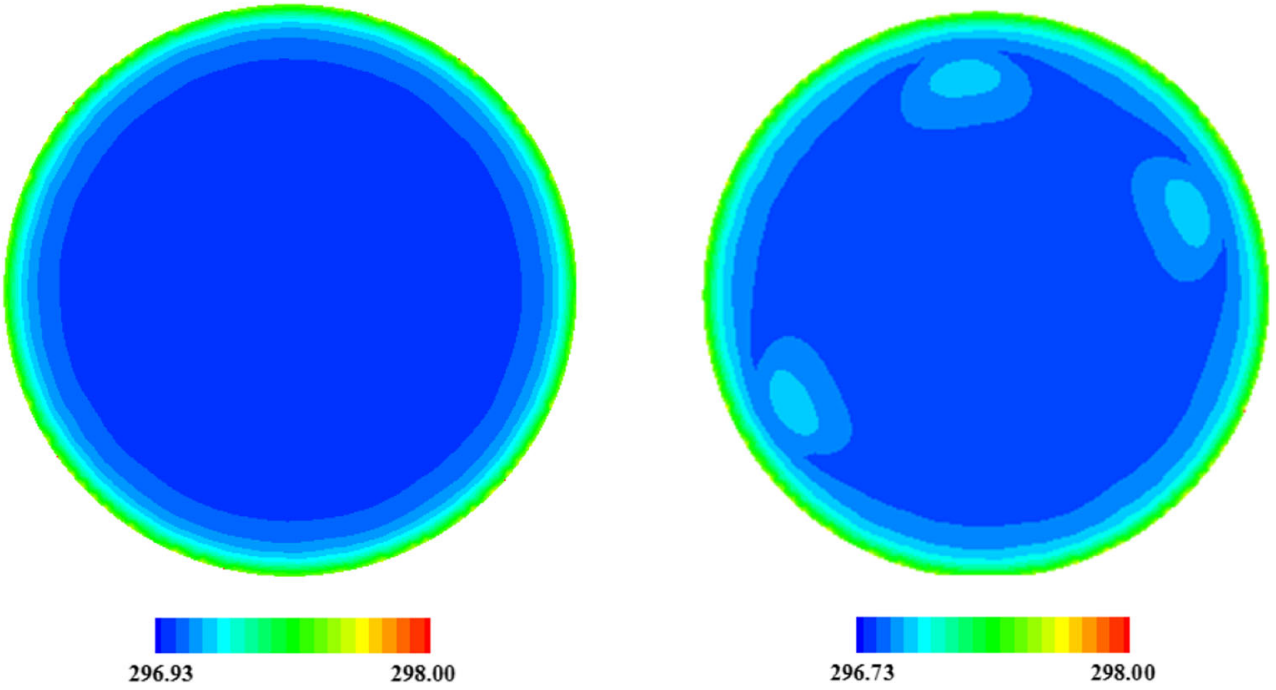

clockwise vortex and a counterclockwise vortex is observed on the inner slice, as shown in Fig. 3. With the further increase of the substrate temperature to $300 \mathrm{~K}$ and $301 \mathrm{~K}$, the fourvortex flow pattern emerges. This variation is thought as the outcome of competition between Marangoni effect and evaporative cooling effect. The evaporation contributes to the decrease of the local temperature. Marangoni convection brings the fluid near the substrate to the free surface, eventually causes the increase of the local temperature on the free surface. The combination of these two basic effects produces a nonlinear temperature along the surface. Accordingly, the surface tension is uneven as well, which generates multicellular flow inside the droplet.

Figure 4 illustrates the variation of the total evaporation mass flux through the droplet surface with the substrate temperatures when pressure ratio is 1 . It is clear that the evaporation mass flux increases when substrate temperature increases. It can be also thought as the consequence of increasing Marangoni flow.

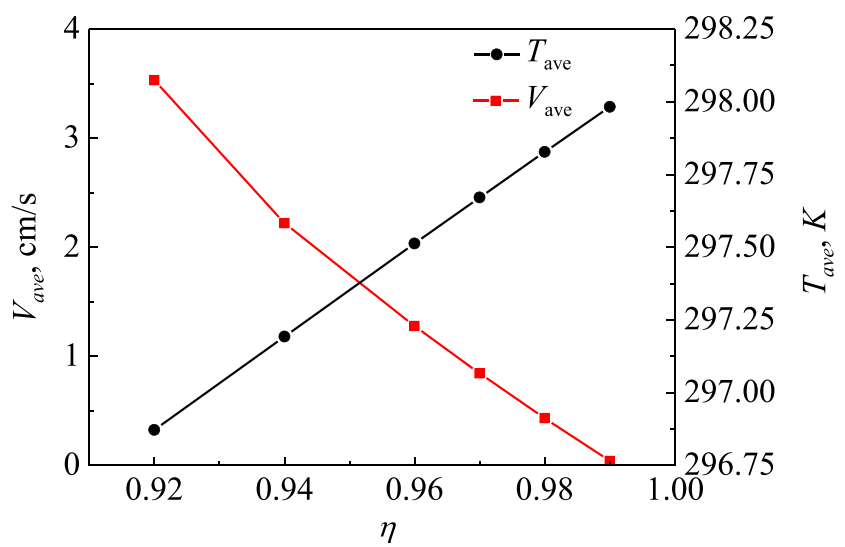

Fig. 7 Variations of average velocity $V_{\text {ave }}$ and average temperature $T_{\text {ave }}$ on the droplet surface with pressure ratio $\eta$

\section{The Influence of Vapor Pressure}

In this section, the substrate temperature is fixed at $298 \mathrm{~K}$, the contact radius and the height of the droplet keep at $r=2.5 \mathrm{~mm}$ and $h=1 \mathrm{~mm}$, respectively. Figure 5 presents the temperature and local evaporation rate along the same curve on droplet surface at different vapor pressure ratios. Obviously, the evaporation rate increases with the decrease of the pressure ratio. Therefore, surface temperature decreases. In addition, the interfacial temperature increases from the droplet center towards the contact line at the edge. The highest temperature appears at the contact line due to the close touching with the isothermal solid substrate. The lowest temperature appears at the apex of the droplet because of the long heat conduction path. Furthermore, the evaporation is rather slight on the most part of the surface, but it becomes dramatic in the region near

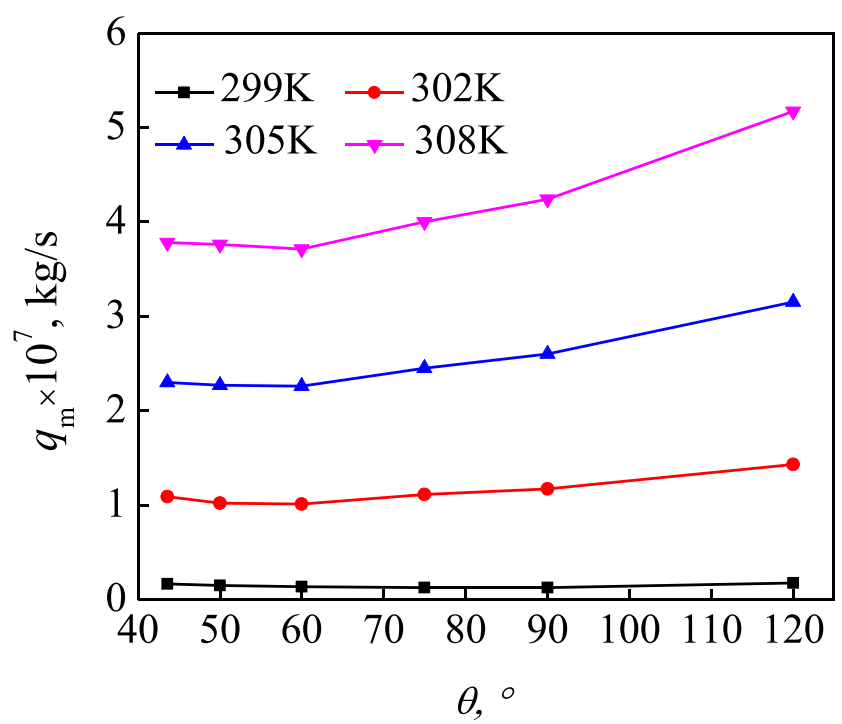

Fig. 8 The variation of total evaporation rate with contact angle at different substrate temperature $(\eta=1)$ 


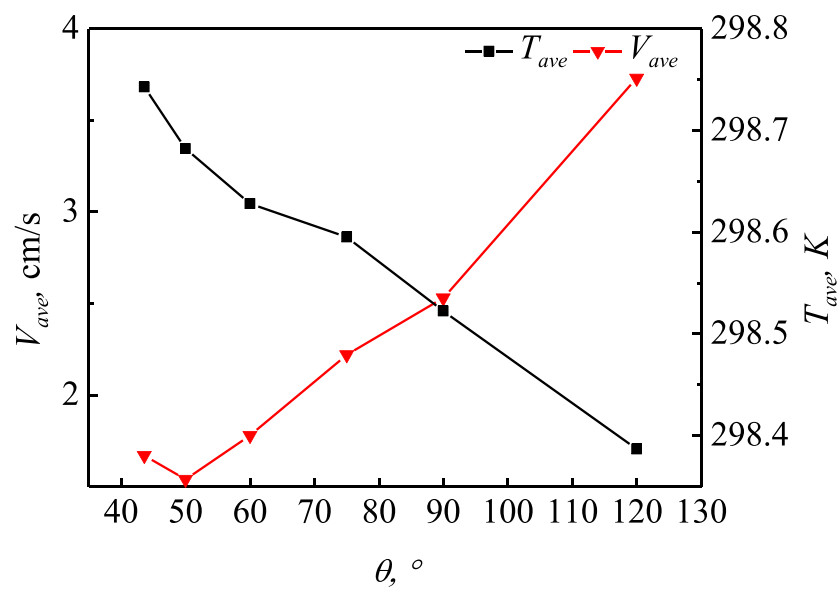

Fig. 9 Variations of the average velocity magnitude $V_{\text {ave }}$ and average temperature $T_{\text {ave }}$ on the droplet surface with the contact angle at $\eta=1$ and $T_{\mathrm{w}}=302 \mathrm{~K}$

the contact line. The temperature on the most part of the surface is close to the temperature of vapor. This trend is similar with the result obtained by Starov and Sefiane (2009) and Nguyen et al. (2010). Nevertheless, in Starov's work, the working liquid is methanol.

Figure 6 presents the variation of surface temperature and flow fields at different pressure ratios. When $\eta \geq 0.93$, the temperature distribution over the surface is ring shape. However, it transits to the triple-cell shape at $\eta \leq 0.92$. This transmission of temperature distribution is directly related to the Marangoni flow. Lower vapor pressure ratio means that less vapor molecules around the droplet, so the resistance for surface evaporation becomes small. With the remarkable evaporation, the vertical temperature gradient is enlarged under the effect of evaporative cooling. Then the Marangoni flow is gradually enhanced because of the temperaturedependent surface tension. This can be inferred from Fig. 7 . The increase of average velocity with pressure ratio indicates the intensifying of Marangoni flow. The average temperature of droplet surface decreases with the pressure ratio because of the reinforced evaporation. In a conclusion, the Marangoni convection becomes dramatic with the decrease of the vapor pressure ratio.

\section{Evaporation at Different Contact Angle}

In this section, $T_{w}=302 \mathrm{~K}, \eta=1$ and $r=2.5 \mathrm{~mm}$. Figure 8 shows the relation between contact angle and corresponding total evaporation mass flux. Obviously, the evaporation mass flux decreases slightly with the increase of contact angle at $\theta$ $<60^{\circ}$. However, when the contact angle is larger than $60^{\circ}$, the trend is reverted. This result is different from the evaporation in the open air (Yang et al. 2014). The variation should be attributed to the relative change of heat conduction path and droplet surface area. When the contact angle is below $60^{\circ}$, the heat conduction path plays a leading role in variation of evaporation flux with contact angle. When the contact angle exceeds $60^{\circ}$, the increase of total evaporation flux versus contact angle is dominantly due to the increase of evaporating surface area. Figure 9 illustrates the variations of average temperature and velocity with contact angle. The decreasing average temperature with contact angle is mainly caused by the increasing heat resistance inside the droplet. The variation of average velocity demonstrates that Marangoni flow becomes remarkable with the increase of the contact angle at $\theta>50^{\circ}$. The velocity magnitude near the contact line is large at big contact angle but is similar in other region, which means Marangoni flow has a more remarkable influence at a big contact angle. To gain a deep insight on the influence of Marangoni flow, we compute the local evaporation rate along the evaporation interface, as shown in Fig.10. It can be inferred that the combined effect of increasing evaporation area and enhancing Marangoni flow devotes to the increase of evaporation flux.

Fig. 10 The local evaporation rate at different contact angle

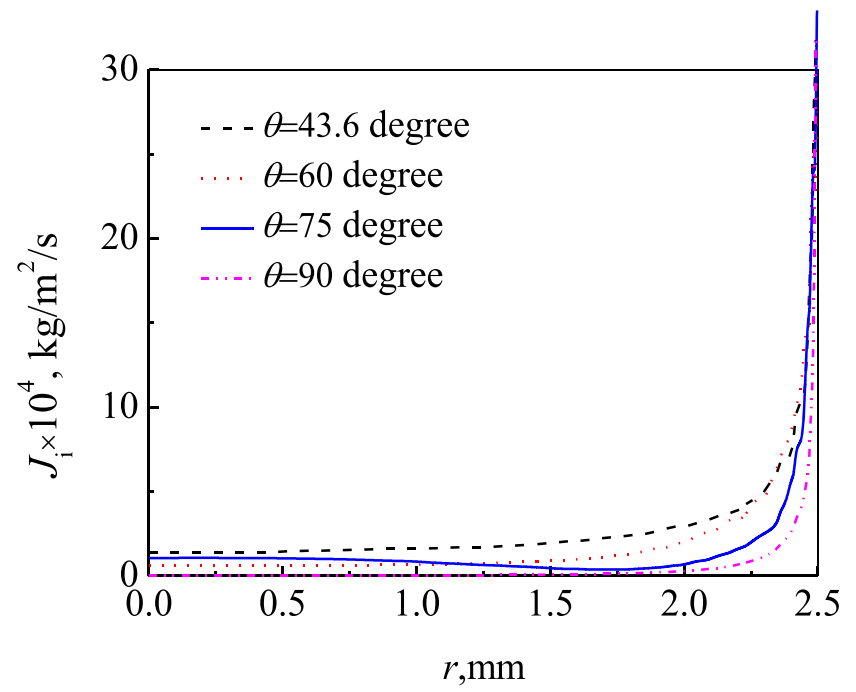




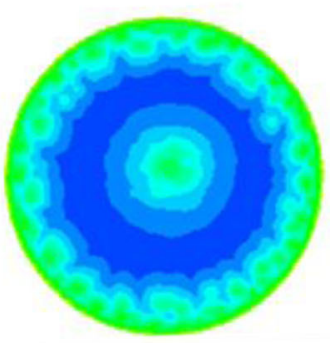

(a) $t=23.54 \mathrm{~s}$

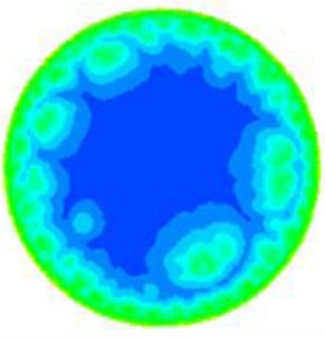

(b) $t=38.54 \mathrm{~s}$

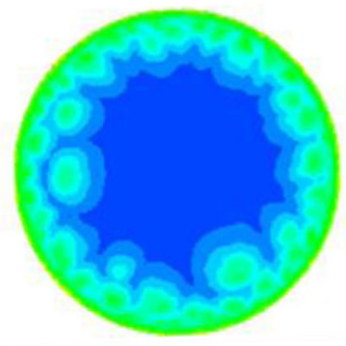

(c) $t=46.06 \mathrm{~s}$

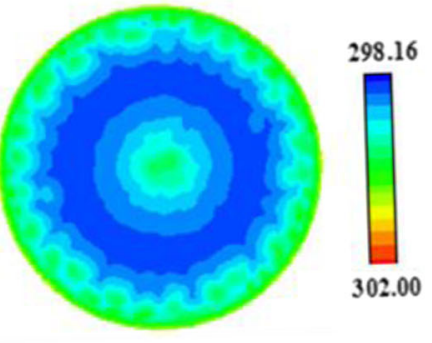

(d) $t=56.04 \mathrm{~s}$

Fig. 11 The evolution of the surface temperature during a period at $\theta=30^{\circ}$

Actually, the vertical temperature and tangential temperature gradients in an evaporating droplet result in different temperature gradient. When the contact angle is above $43.6^{\circ}$, the temperature distribution along the free surface is smooth. The transition for the dominant influences from tangential to vertical temperature gradient happens at $\theta=43.6^{\circ}$.

When the contact angle decreases to $30^{\circ}$, the flow transits the time-dependent oscillatory flow and the surface temperature begins to oscillate. Figure 11 shows the temperature evolution on the droplet surface during a period. This phenomenon can be explained by the combining effects of Marangoni convection and evaporation. At this specific contact angle, these two effects are equally competing with each other, which results in the appearance of the stagnant point. Compared with droplet of large contact angle, the droplet evaporation at a small contact angle is more remarkable because much energy is supplied from the substrate. Strong evaporation decreases the surface temperature dramatically. The temperature gradient along the interface is enlarged owing to notable evaporation and contributes to strengthen Marangoni convection. The enhanced Marangoni flow brings the hot fluid near the substrate to droplet surface and devotes to the heat transfer inside the droplet. The reduced temperature caused by evaporation is increased again by Marangoni effect
Fig. 12 The temperature

variation at a monitor $(x=0, y=0$, $z=0.67 \mathrm{~mm}$ ) when $\theta=30^{\circ}$

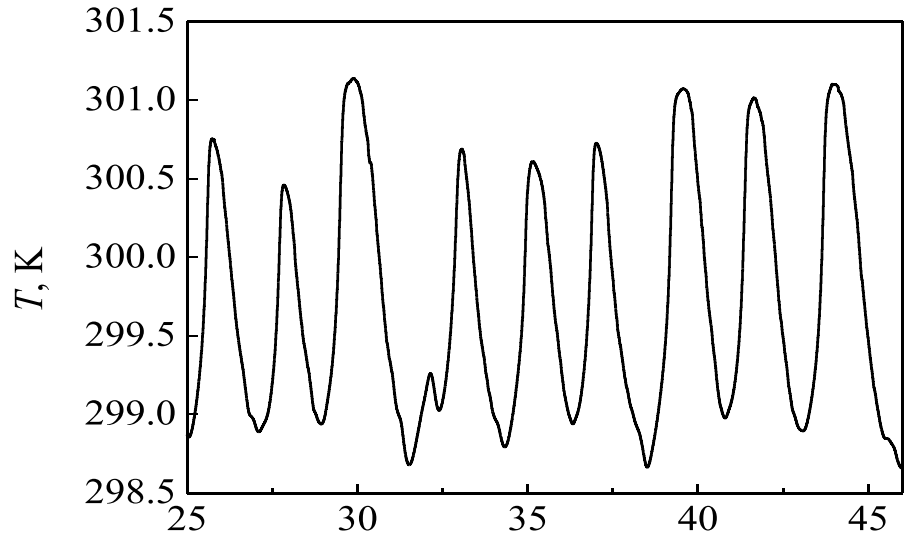

Fig. 13 Snapshots (left) of surface and inner slice temperature distribution and the temperature distribution (right) along the curve $\mathrm{M}$ on the droplet surface at $\theta=9.15^{\circ}$
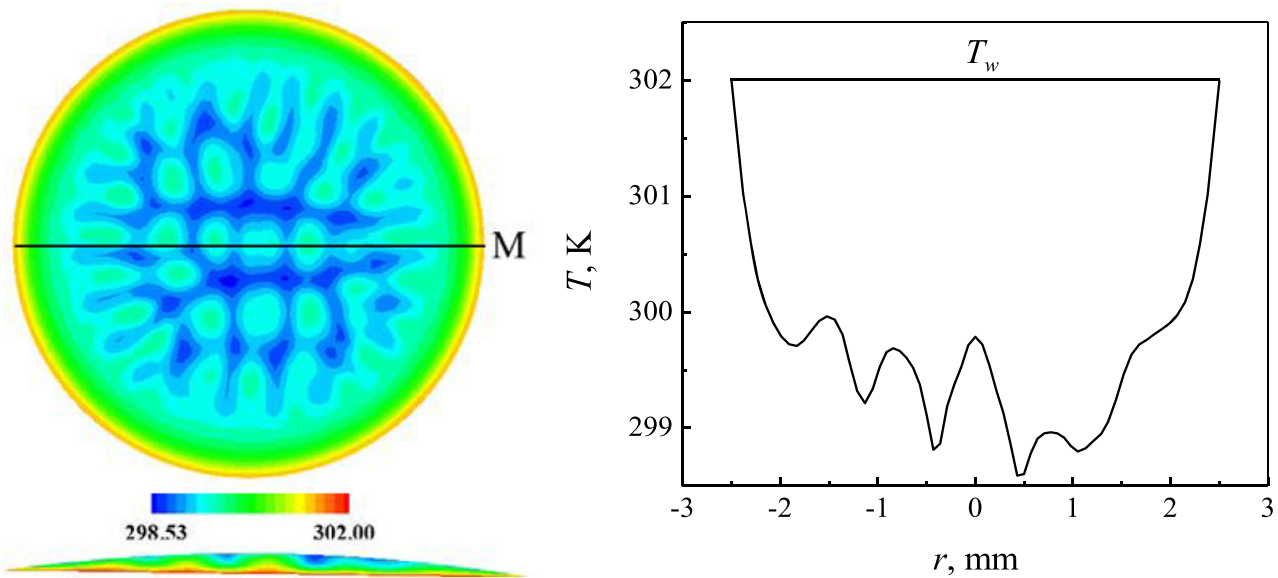
and contributes to prominent evaporation with the help of Marangoni flow, which finally leads to the oscillation of temperature. This can be evidenced by temperature variation at a monitor ( $x=0, y=0, z=0.67 \mathrm{~mm}$ ), as plotted in Fig. 12 .

When contact angle decreases to $\theta=9.15^{\circ}$, the unstable multiple-cell temperature distribution is observed on the droplet surface, as shown in Figure 13. This flow pattern formation should be attributed to the short heat conduction path. In the area near the contact line, the heat loss caused by evaporation can be supplemented instantly by heat conduction because the heat resistance is small. Compared with the peripheral region, the top area on the droplet surface is far from the substrate. The relative long heat conduction path and evaporation lead to the low temperature of this area. Therefore, the large vertical temperature gradient generates, which results in the strong Marangoni-Benard flow. The influence of the MarangoniBenard flow on the temperature is larger than the evaporative cooling, so the non-monotonic temperature distribution in the middle region of droplet surface is finally formed. Figure 13 illustrates the temperature distribution along the interface. It shows that non-monotonic temperature mainly appears on the top region of the droplet, which is regarded as the effect of Marangoni-Benard convection. Both Chen et al. (2015) and Bouchenna et al. (2015) found multi-cellular pattern inside the droplet at a small contact angle by using a two-dimension model. But they only obtained the flow pattern inside the droplet.

\section{Conclusions}

The effects of the substrate temperature, vapor pressure ratio and contact angle on a sessile water droplet evaporation are investigated numerically. According to the simulation results, several conclusions are obtained:

(1) Increasing substrate temperature contributes to the intensifying evaporation and results in different flow patterns by strong Marangoni flow. The temperature distribution on the droplet surface converts from ring shape, petal shape to serrated shape with the increase of substrate temperature.

(2) The decrease of pressure ratio devotes to the increase of total evaporation flux and transmission of flow pattern. There is an abrupt change of the flow and temperature fields at $\eta=0.92$. The enhancing evaporation could facilitate Marangoni convection with decrease of pressure ratio.

(3) The surface flow pattern tends to be complex at a small contact angle. When the contact angle is below $30^{\circ}$, the temperature distribution over the droplet varies regularly. The multi-cellular temperature distribution is observed on the liquid-vapor interface at $\theta=9.15^{\circ}$. Furthermore, the relation between total corresponding evaporation flux and contact angle is non-monotonic.

Acknowledgments This work is supported by National Natural Science Foundation of China (Grant No. 11532015), Chongqing University Postgraduates Innovation Project (Grant No: CYS18041) and the Fundamental Research Funds for the Central Universities (No. 2018CDXYDL0001).

Publisher's Note Springer Nature remains neutral with regard to jurisdictional claims in published maps and institutional affiliations.

\section{References}

Barash, L.Y.: Marangoni convection in an evaporating droplet: analytical and numerical descriptions. Int. J. Heat Mass Transf. 102, 445-454 (2016)

Bouchenna, C., Saada, M.A., Chikh, S., Tadrist, L.: Investigation of thermo-capillary flow inside an evaporating pinned water droplet. Interfacial Phenomena and Heat Transfer. 3(2), 185-201 (2015)

Brutin, D., Zhu, Z.Q., Rahli, O., Xie, J.C., Liu, Q.S., Tadrist, L.: Evaporation of ethanol drops on a heated substrate under microgravity conditions. Microgravity Sci. Technol. 22(3), 387-395 (2010)

Chen, X., Zhu, Z.Q., Liu, Q.S., Wang, X.W.: Thermodynamic behaviors of macroscopic liquid droplets evaporation from heated substrates. Microgravity Sci. Technol. 27(5), 353-360 (2015)

Chen, X., Wang, X., Chen, P.G., Liu, Q.S.: Thermal effects of substrate on Marangoni flow in droplet evaporation: response surface and sensitivity analysis. Int. J. Heat Mass Transf. 113, 354-365 (2017)

Chen, X., Wang, X., Chen, P.G., Liu, Q.S.: Determination of diffusion coefficient in droplet evaporation experiment using response surface method. Microgravity Sci. Technol. 30(5), 675-682 (2018)

Cheng, W.L., Han, F.Y., Liu, Q.N., Zhao, R., Fan, H.L.: Experimental and theoretical investigation of surface temperature non-uniformity of spray cooling. Energy. 36(1), 249-257 (2011)

Cheng, W.L., Zhang, W.W., Chen, H., Hu, L.: Spray cooling and flash evaporation cooling: the current development and application. Renew. Sust. Energ. Rev. 55, 614-628 (2016)

Crafton, E.F., Black, W.Z.: Heat transfer and evaporation rates of small liquid droplets on heated horizontal surfaces. Int. J. Heat Mass Transf. 47(6), 1187-1200 (2004)

D'Agaro, P., Croce, G., Cortella, G.: Numerical simulation of glass doors fogging and defogging in refrigerated display cabinets. Appl. Therm. Eng. 26(16), 1927-1934 (2006)

Deegan, R.D., Bakajin, O., Dupont, T.F., Huber, G., Nagel, S.R., Written, T.A.: Capillary flow as the cause of ring stains from dried liquid drops. Nature. 389(6653), 827-829 (1997)

Dunn, G.J., Wilson, S.K., Duffy, B.R., David, S., Sefiane, K.: The strong influence of substrate conductivity on droplet evaporation. J. Fluid Mech. 623(623), 329-351 (2009a)

Dunn, G.J., Wilson, S.K., Duffy, B.R., Sefiane, K.: Evaporation of a thin droplet on a thin substrate with a high thermal resistance. Phys. Fluids. 21(5), 336-181 (2009b)

Ghasemi, H., Ward, C.A.: Energy transport by thermocapillary convection during sessile-water-droplet evaporation. Phys. Rev. Lett. 105(13), $136102(2010)$

Girard, F., Antoni, M.: Influence of substrate heating on the evaporation dynamics of pinned water droplets. Langmuir 24(20), 11342 (2008), 11345

Girard, F., Antoni, M., Faure, S., Steinchen, A.: Numerical study of the evaporating dynamics of a sessile water droplet. Microgravity Sci. Technol. 18(3-4), 42-46 (2006) 
Girard, F., Antoni, M., Faure, S., Steinchen, A.: Influence of heating temperature and relative humidity in the evaporation of pinned droplets. Colloid Surf. A-Physicochem. Eng. Asp. 323(1-3), 36-49 (2008)

Haschke, T., Wiechert, W., Graf, K., Bonaccurso, E., Li, G., Suttmeier, F.T.: Evaporation of solvent microdrops on polymer substrates: from well controlled experiments to mathematical models and back. Nanoscale Microscale Thermophys. Eng. 11(1-2), 31-41 (2007)

Hu, H., Larson, R.G.: Evaporation of a sessile droplet on a substrate. J. Phys. Chem. B. 106(6), 1334-1344 (2002)

$\mathrm{Hu}, \mathrm{H}$., Larson, R.G.: Analysis of the microfluid flow in an evaporating sessile droplet. Langmuir. 21(9), 3963-3971 (2005)

Kelly, P.L.: Vapor distribution above an evaporating sessile drop. Int. J. Heat Mass Transf. 65, 165-172 (2013)

Liu, L., Mi, M.L.: Theoretical investigation on rapid evaporation of a saline droplet during depressurization. Microgravity Sci. Technol. 25(5), 295-302 (2014)

Marek, R., Straub, J.: Analysis of the evaporation coefficient and the condensation coefficient of water. Int. J. Heat Mass Transf. 44(1), 39-53 (2001)

Nguyen, T.A.H., Nguyen, A.V., Hampton, M.A., Xu, Z.P., Huang, L.B., Rudolph, V.: Theoretical and experimental analysis of droplet evaporation on solid surfaces. Chem. Eng. J. 69(1), 522-529 (2010)

Park, J., Moon, J.: Control of colloidal particle deposit patterns within picoliter droplets ejected by ink-jet printing. Langmuir. 22(8), 35063513 (2006)

Pericet-Camara, R., Bonaccurso, E., Graf, K.: Microstructuring of polystyrene surfaces with nonsolvent sessile droplets. Chem. Phys. Chem. 9(12), 1738-1746 (2010)

Persad, A.H., Ward, C.A.: Expressions for the evaporation and condensation coefficients in the hertz-Knudsen relation. Chem. Rev. 116(14), 7727-7767 (2016)

Picknett, R.G., Bexon, R.: Evaporation of sessile or pendant drops in still air. J. Colloid Interface Sci. 61(2), 336-350 (1977)

Sefiane, K.: On the formation of regular patterns from drying droplets and their potential use for bio-medical applications. J. Bionic Eng. 7(4), S82-S93 (2010)

Sefiane, K., Bennacer, R.: An expression for droplet evaporation incorporating thermal effects. J. Fluid Mech. 667(1), 260-271 (2011)
Sefiane, K., David, S., Dunn, G.: Analysis of the diffusion model for sessile drops evaporating under reduced pressure and the influence of the properties of the substrate and ambient atmosphere. Epigenetics. 7(10), 1125-1132 (2007)

Semenov, S., Starov, V.M., Rubio, R.G., Agogo, H., Velarde, M.G.: Evaporation of sessile water droplets: universal behaviour in presence of contact angle hysteresis. Colloid Surf. A-Physicochem. Eng. Asp. 391(1-3), 135-144 (2012a)

Semenov, S., Starov, V.M., Rubio, R.G., Velarde, M.G.: Computer simulations of evaporation of pinned sessile droplets: influence of kinetic effects. Langmuir. 28(43), 15203-15211 (2012b)

Semenov, S., Carle, F., Medale, M., Brutin, D.: 3D unsteady computations of evaporative instabilities in a sessile drop of ethanol on a heated substrate. Appl. Phys. Lett. 111(24), 241602 (2017)

Sodtke, C., Stephan, P.: Spray cooling on micro structured surfaces. Int. J. Heat Mass Transf. 50(19), 4089-4097 (2007)

Starov, V., Sefiane, K.: On evaporation rate and interfacial temperature of volatile sessile drops. Colloid Surf. A-Physicochem. Eng. Asp. 333(1), 170-174 (2009)

Unsworth, M.H.: Principles of environmental physics. Phys. Today. 5(2), 66 (1974)

Wang, Y.L., Ma, L.R., Xu, X.F., Luo, J.B.: Expressions for the evaporation of sessile liquid droplets incorporating the evaporative cooling effect. J. Colloid Interface Sci. 484, 291-297 (2016)

$\mathrm{Xu}$, X.F., Luo, J.B.: Marangoni flow in an evaporating water droplet. Appl. Phys. Lett. 91(12), 3972 (2007)

$\mathrm{Xu}$, X.F., Ma, L.R.: Analysis of the effects of evaporative cooling on the evaporation of liquid droplets using a combined field approach. Soft Matter. 5(28), 8614 (2015)

Yang, K., Hong, F.J., Cheng, P.: A fully coupled numerical simulation of sessile droplet evaporation using arbitrary Lagrangian-Eulerian formulation. Int. J. Heat Mass Transf. 70(3), 409-420 (2014)

Ye, S., Wu, C.M., Zhang, L., Li, Y.R., Liu, Q.S.: Evolution of thermal patterns during steady state evaporation of sessile droplets. Exp. Thermal Fluid Sci. 98, 712-718 (2018)

Zhu, Z.Q., Brutin, D., Liu, Q.S., Wang, Y., Mourembles, A., Xie, J.C., Tadrist, L.: Experimental investigation of pendant and sessile drop in microgravity. Microgravity Sci. Technol. 22(3), 339-345 (2010) 